\title{
Transtorno de ansiedade social e habilidades sociais de falar em público: estudo experimental
}

\section{Social anxiety disorder and social skills for speaking in public: experimental study}

\author{
Antonio Paulo Angélico* \\ Universidade Federal de São João del-Rei - UFSJ, São João del-Rei, Minas Gerais, \\ Brasil
}

\section{José Alexandre S. Crippa**}

Universidade de São Paulo - USP, Ribeirão Preto, São Paulo, Brasil

\section{Sonia Regina Loureiro***}

Universidade de São Paulo - USP, Ribeirão Preto, São Paulo, Brasil

\begin{abstract}
RESUMO
O falar em público tem sido considerado um importante estressor psicossocial. Objetivou-se, comparar e caracterizar o repertório de habilidades sociais apresentado por universitários portadores de Transtorno de Ansiedade Social (TAS) e não portadores frente a uma situação experimental estruturada, o Teste de Simulação de Falar em Público (TSFP). Participaram 26 universitários, sendo 13 com diagnóstico de TAS (grupo caso de TAS) e 13 não portadores de transtornos psiquiátricos (grupo nãocaso). Esses grupos não apresentaram diferenças significativas para a maioria dos marcadores comportamentais de ansiedade. Um número maior de sujeitos do grupo não-caso foi avaliado como apresentando um nível de habilidades sociais apropriadas para falar em público, em comparação ao grupo caso. Os grupos diferiram significativamente na maioria dos itens indicativos da habilidade de falar em público do Inventário de Habilidades Sociais (IHS-Del-Prette). Considera-se que a avaliação por meio do TSFP pode favorecer o planejamento de programas de treinamento em habilidades sociais.
\end{abstract}

Palavras-chave: Transtorno de ansiedade social, falar em público, habilidades sociais, ansiedade, universitários.

\footnotetext{
ABSTRACT

Speaking in public is considered an important psychosocial stressor. The objective of this investigation was to compare and characterize the repertoire of social skills displayed by college students suffering from Social Anxiety Disorder (SAD) and non-cases in a structured experimental situation, Simulation of Public Speaking Test (SPST). 26 undergraduate students participated, 13 of whom were diagnosed with SAD, and 13 noncases of any psychiatric disorder (non-case group). These groups showed no significant differences for most behavioral markers of anxiety. A larger number of subjects in the non-case group were assessed as having a level of
} 
appropriate social skills for speaking in public, in comparison with the case group. The groups differed significantly on most items indicative of the ability to speak in public of the Social Skills Inventory (IHS-Del-Prette). It is considered that the assessment through SPST may facilitate the planning of social skills training programs.

Keywords: Social anxiety disorder, public speaking, social skills, anxiety, college students.

\section{I ntrodução}

O falar em público tem sido considerado um importante estressor psicossocial (D'EL REY; PACINI, 2005; GIRDER et al., 1990; SAAB et al., 1989), eliciando grande ansiedade e afetos negativos, além de respostas neuroendócrinas, metabólicas, imunológicas, cardiovasculares e eletrodérmicas (GONZALEZ-BONO et al., 2002). Segundo D'El Rey e Pacini (2005), o medo acentuado de falar em público deve ser encarado como um problema sério de saúde mental por interferir na rotina de trabalho, na vida social e na vida escolar, produzindo, assim, sofrimento aos indivíduos acometidos por ele.

Vários autores têm apontado a alta prevalência do medo de falar em público, tanto entre os indivíduos portadores do transtorno de ansiedade social (TAS), como também na população geral (FURMARK et al., 1999; FURMARK et al., 2000; GEER, 1965; MANNUZZA et al., 1995; OSÓRIO; CRIPPA; LOUREIRO, 2008a, 2008b; STEI N; WALKER; FORDE, 1996). Este fato tem despertado e estimulado estudos que avaliem e dimensionem esta situação específica.

Em 1982, McNair et al. desenvolveram e validaram um modelo para avaliação da ansiedade, nomeado de "Teste de Simulação de Falar em Público - TSFP", que consiste em solicitar ao sujeito que prepare um discurso e o fale em frente a uma câmera de vídeo, que estará gravando sua performance. Guimarães, Zuardi e Graeff (1988) modificaram esse modelo original, introduzindo juntamente às medidas fisiológicas avaliadas durante o procedimento, tais como: pressão arterial, batimentos cardíacos e condutância da pele, medidas relacionadas aos estados subjetivos experimentados. Para tal propuseram o uso do Inventário de Ansiedade Traço-Estado de Spielberger (IDATE; SPIELBERGER; GORSUCH; LUSHINE, 1970) e a Escala Analógica de Humor (VAMS - traduzida e adaptada para o português por ZUARDI; KARNIOL, 1981).

Segundo Graeff et al. (2003), este modelo modificado tem sido amplamente utilizado em estudos farmacológicos, e também em estudos relativos a outros transtornos de ansiedade, a exemplo do pânico (HETEM et al., 1996).

Rapee e Lim (1992) realizaram um estudo visando verificar como indivíduos com e sem TAS avaliam seus desempenhos por meio de uma situação simulada de falar para uma pequena audiência e de 
uma bateria de instrumentos específicos de auto e hetero-avaliação do comportamento e dos estados subjetivos. Encontraram que, para todos os sujeitos, a auto-avaliação foi discrepante, especialmente quando comparada à hetero-avaliação. Porém, nos indivíduos com TAS, esta discrepância foi muito mais significativa, apontando para a importância do componente cognitivo do transtorno.

A prevalência, o impacto e o funcionamento dos indivíduos com medo de falar em público foram estudados por Stein, Walker e Forde (1996), tendo como participantes indivíduos da população geral. Identificaram que $57 \%$ do total dos participantes relataram medo incluído nas classificações "significativo" até "bastante significativo". Estes participantes comparativamente aos demais, cujo medo não foi classificado como significativo, apresentaram menor nível educacional, nível econômico mais baixo e menor renda pessoal, destacando assim importantes prejuízos de ordem psicossocial associados ao temor de falar em público.

Em outro estudo, Hofmann et al. (1997) objetivaram avaliar se haviam diferenças entre os indivíduos com TAS e controles e entre os subtipos generalizado e circunscrito do TAS quanto aos distúrbios da fala (exemplificados como pausas, repetições, sentenças incompletas, gagueira, sons incoerentes) e quanto à dificuldade em manter o contato visual na situação de falar em público, uma vez que tais aspectos parecem ser bastante característicos do medo social. Os resultados apontaram que os indivíduos com TAS apresentam maior nível de ansiedade durante o falar em público, comparativamente aos controles. Observaram que os indivíduos com TAS apresentam falas com pausas mais longas e em maior quantidade, comparativamente aos sem TAS, apontando para a validade deste comportamento como um indicador real do medo social. O mesmo não ocorreu com o comportamento de fixar o olhar, pois não se observou diferenças quanto aos diversos grupos estudados em relação à quantidade e duração do contato visual durante a situação experimental.

Destacam-se ainda outros estudos que avaliaram aspectos diversos relacionados ao falar em público, tais como os relativos ao funcionamento cerebral (TILLFORS et al., 2002), a utilização de recursos virtuais como modelo terapêutico (HARRIS; KEMMERLING; NORTH, 2002; LEE et al., 2002a; LEE et al., 2002b; PERTAUB; SLATER; BARKER, 2001) e a avaliação das características psicométricas de instrumento específico para avaliação do falar em público (PHILLIPS et al., 1997). Este estudo psicométrico realizou a normatização em amostra de indivíduos universitários do Personal Report of Confidence as a Speaker, um instrumento de rastreamento, composto por 30 itens avaliados como falso ou verdadeiro. A validade discriminativa deste instrumento mostrou-se adequada, revelando que os níveis de ansiedade experimentados ao falar em público são 
razoavelmente constantes, considerando-se o gênero, a etnia e a idade (PHILLIPS et al., 1997).

Tendo em vista que o desempenho de falar em público pode constituir uma importante medida ou estratégia de avaliação clínica do TAS, enquanto uma habilidade componente do repertório interpessoal global dos indivíduos, torna-se fundamental dispor de instrumentos validados e abrangentes que avaliem tanto os recursos e déficits comportamentais quanto os prejuízos sociais e funcionais destes indivíduos, visando sistematizar procedimentos de diagnóstico mais precisos que possibilitem a implementação de medidas de intervenção, atendendo de modo mais direto, apropriado e objetivo as suas necessidades pessoais e profissionais.

Assim sendo, a avaliação do desempenho social, especialmente no que se refere ao falar em público dos estudantes universitários, assume inquestionável relevância tanto social quanto educacional, considerando-se o elevado comprometimento social e funcional, evidenciado na qualidade de vida de indivíduos com TAS; e também pelo fato dos estudantes universitários constituírem uma classe profissional emergente, cuja interação social é a sua base de ação profissional, pelo menos em algumas áreas do Conhecimento, quando não também o objeto e objetivo dessa atuação (DEL PRETTE; DEL PRETTE; CASTELO BRANCO, 1992a; DEL PRETTE; DEL PRETTE; CASTELO BRANCO, 1992b).

Para um melhor desempenho acadêmico e social dos universitários, pode ser considerado imprescindível a presença de um repertório de habilidades interpessoais, tais como: aproximar-se de outras pessoas, iniciar contato e conversação, estabelecer contato visual, defender os próprios direitos, criticar, fazer pedidos, recusar pedidos abusivos, pedir mudança de comportamento, entre outras. Além dessas habilidades, o desempenho de falar em público deve também ocorrer por meio de indicadores sociolinguísticos, tais como: a construção da fala, a pronúncia, a concentração no conteúdo da fala, a consciência do outro na interação e a autoconsciência pública.

\section{Objetivo}

Tem-se por objetivo comparar e caracterizar o repertório de habilidades sociais, apresentado por estudantes universitários, portadores de TAS e não portadores, frente a uma situação experimental estruturada, denominada Teste de Simulação de Falar em Público (TSFP). 


\section{Método}

\subsection{Sujeitos}

Participaram neste estudo 26 alunos universitários, provenientes da Faculdade de Medicina de Ribeirão Preto (FMRP), e da Faculdade de Filosofia, Ciências e Letras de Ribeirão Preto (FFCLRP) da Universidade de São Paulo (USP), selecionados a partir da amostra do estudo maior, do qual este faz parte.

Para a composição da amostra deste estudo, os nomes de 135 sujeitos do estudo maior foram alocados randomicamente em dois grupos, a saber:

- Caso: composto por 64 sujeitos que obtiveram resposta positiva na escala de auto-avaliação Mini-SPIN (mínimo de seis pontos nos três itens - 6, 9, 15 -, que a compõem) e com diagnóstico clínico de TAS, avaliados por meio da Entrevista Clínica Estruturada para o DSM-IV (SCID);

- Não-caso: constituído por 71 sujeitos que obtiveram resposta negativa na Mini-SPIN (zero a um ponto nos itens que a compõem) e sem diagnóstico clínico de TAS.

Uma tabela de números aleatórios foi utilizada para a designação dos sujeitos aos grupos. Posteriormente, adotaram-se os seguintes critérios de inclusão: localização dos sujeitos, disponibilidade para participar do estudo e para comparecer ao Hospital das Clínicas (HC) da FMRP da USP em dia e horário previamente agendado para a realização das atividades previstas, e (c) ter respondido ao IHS-DelPrette. Foram excluídos aqueles sujeitos que apresentassem as seguintes comorbidades ou condições psiquiátricas: (a) quadros psicóticos, (b) depressão atual, (c) depressão recorrente, (d) transtorno alimentar atual, (e) abuso de substâncias atual, (f) transtorno obsessivo-compulsivo atual, (g) dependência de substâncias atual, (h) episódio hipomaníaco, (i) transtorno do pânico, e (j) TAS subclínico (sujeitos que apresentassem medo não razoável de uma situação social, mas que não preenchiam os critérios de evitação ou prejuízo funcional e/ou ocupacional devido a este medo). Estabeleceu-se como meta avaliar de 25 a 30 participantes e, aplicando-se sistematicamente os critérios de exclusão, foi obtida uma amostra de 26 estudantes universitários, entre 19 e 25 anos, de ambos os gêneros, independentemente do nível socioeconômico, matriculados em diversos cursos de graduação oferecidos no campus de Ribeirão Preto da Universidade de São Paulo, e que aceitaram participar do estudo, assinando o Termo de Consentimento Livre e Esclarecido (TCLE) (CEP - Processo HCRP №. 6064/2006). Os sujeitos deste estudo foram alocados em dois grupos, caso e não-caso, por 
pareamento quanto ao gênero e número de indivíduos com e sem diagnóstico de TAS.

\subsection{I nstrumentos e equipamentos}

No processo de seleção e identificação dos sujeitos, que constituíram os dois grupos, foram utilizados os seguintes instrumentos:

- Mini-SPIN - escala auto-aplicável, composta por três itens ("Evito fazer coisas ou falar com certas pessoas por medo de ficar envergonhado", "Evito atividades nas quais sou o centro das atenções", "Ficar envergonhado ou parecer bobo são meus maiores temores"), avaliados em uma escala de cinco pontos. Usando um escore de corte igual ou maior que seis, a Mini-SPIN demonstrou possuir boas propriedades psicométricas: uma sensibilidade de $88,7 \%$, especificidade de $90,0 \%$, valor preditivo positivo de $52,6 \%$ e valor preditivo negativo de $98,5 \%$, e $89,9 \%$ de precisão no diagnóstico do TAS (CONNOR et al., 2001).

- Entrevista Clínica Estruturada para o DSM-IV (SCID-CV - versão clínica; DEL-BEN et al., 2001a) - instrumento utilizado para a elaboração de diagnósticos clínicos psiquiátricos baseados no DSM-IV. É composto por módulos, em um total de dez, que podem ser aplicados de forma independente ou combinada, conforme os objetivos almejados. Neste estudo, foram utilizados o Módulo de Ansiedade (Módulo F) e o Critério A de cada um dos outros módulos, durante uma entrevista telefônica, visando à confirmação diagnóstica do TAS e a identificação da presença de comorbidades. Posteriormente, o Módulo $F$ foi aplicado novamente durante uma entrevista individual "ao vivo". No estudo maior, do qual este faz parte, entrevistas realizadas com 100 sujeitos, primeiramente por telefone e depois pessoalmente, demonstraram uma concordância excelente, com um coeficiente kappa de 0,84, sugerindo que ambas formas de diagnóstico são comparáveis (CRIPPA et al., 2008).

- Inventário de Habilidades Sociais (IHS-Del-Prette) - instrumento de autorrelato para a avaliação das dimensões situacional e comportamental molar das habilidades sociais, contendo 38 itens, cada um deles descrevendo uma relação interpessoal e uma possível reação àquela situação. Cada item pode ser classificado de 0 (nunca ou raramente) até 4 (sempre ou quase sempre), com escore total variando de 0 a 152. A análise das propriedades psicométricas, no estudo original, junto a 527 universitários obteve os seguintes resultados: índices de discriminação positivos, variando de 3,0 a 16,7; consistência interna satisfatória com um alfa de Cronbach de 0,75; e estrutura fatorial com cinco fatores principais, identificados em termos comportamentais-situacionais, com coeficientes alfa variando de 0,74 a 0,96 , que explicam $92,75 \%$ da variância total (DEL PRETTE; DEL PRETTE; BARRETO, 1998). 
- Teste de Simulação de Falar em Público (TSFP): desenvolvido por McNair et al. (1982) e modificado por Guimarães, Zuardi e Graeff (1988), o teste consiste em solicitar ao sujeito que prepare um discurso e depois o apresente em frente a uma câmara de vídeo que estará gravando o seu desempenho.

Durante o período de coleta de dados, além destes instrumentos, foram utilizados os seguintes equipamentos e recursos: filmadora, fitas de vídeo, televisão, e vídeo cassete. Para a análise dos dados, foram empregados: televisão, DVD Player, DVD's com as filmagens do TSFP, e um Protocolo de Registro das Filmagens Realizadas.

\subsection{Treinamento de avaliadores externos}

Dois alunos de graduação em Psicologia foram treinados pelo primeiro autor para procederem à análise independente de 10 sessões de observação (38,5\% do total), visando o cálculo do índice de concordância entre eles. Cada avaliador externo analisou o conteúdo das filmagens de cinco sujeitos, selecionadas randomicamente por meio de sorteio. Durante o treinamento, os índices de concordância obtidos entre o pesquisador e cada um dos avaliadores foram de 96,0 e $94,8 \%$, respectivamente.

\subsection{Procedimentos}

Os procedimentos empregados para a coleta e análise de dados deste estudo serão descritos a seguir.

\subsubsection{Coleta de dados}

Os sujeitos selecionados para participar deste estudo foram previamente contatados por telefone por um psiquiatra e avaliados quanto ao Módulo de Ansiedade (Módulo F) e Critério A da SCID-IV, objetivando verificar a ausência ou presença de comorbidades. Aos sujeitos que não apresentaram comorbidades psiquiátricas, consideradas pelos critérios de exclusão adotados, foi perguntado sobre a disponibilidade de continuar participando do estudo. Aos que concordaram, solicitou-se comparecer no HC da FMRP-USP, em dia e horário previamente agendado de acordo com a disponibilidade individual, para participarem de uma entrevista individual para confirmação diagnóstica e, posteriormente, de uma situação experimental considerada um teste de ansiedade. O tempo de duração das entrevistas individuais "ao vivo" foi em média de três a cinco minutos. Na ocasião da entrevista, os sujeitos preencheram o IHS-Del-Prette e outros instrumentos que foram objetos de estudo das demais pesquisas vinculadas ao projeto maior (CEP - Processo HCRP no. 11570/2003). 
A aplicação do TSFP foi realizada por duas assistentes de pesquisa, a saber: uma psiquiatra, previamente treinada, e uma técnica de laboratório com ampla experiência na aplicação do procedimento, já consolidada em estudos prévios (CHAVES, 2007; DEL-BEN et al., 2001b; GUIMARÃES; MBAYA; DEAKIN, 1997; ZUARDI et al., 1993). No dia agendado para o teste, explicaram-se os objetivos do teste a ser realizado e somente prosseguiu-se com aqueles sujeitos que devidamente assinaram o TCLE.

Depois, pediu-se ao sujeito que aguardasse por um período de 30 minutos na própria sala, permanecendo deitado, procurando relaxarse. Foram oferecidas revistas com conteúdo neutro ("Caras", "Quem", "Contigo") com este propósito e a assistente de pesquisa retirou-se da sala. Após o término do período, a assistente de pesquisa retornava à sala para iniciar, então, o procedimento do TSFP, conforme proposto por Guimarães, Zuardi e Graeff (1988).

A sessão experimental foi conduzida em uma sala com atenuação sonora e temperatura controlada, conforme procedimento proposto por Guimarães, Zuardi e Graeff (1988). O sujeito sentava-se em frente a uma câmera de vídeo e assistia a um vídeotape pré-gravado com as seguintes instruções sobre a tarefa que teria que desempenhar: "Você terá dois minutos para preparar um discurso, de quatro minutos, sobre o sistema de transporte de sua cidade, que será gravado e, posteriormente, analisado por um psicólogo". Foi computado o tempo de dois minutos para a elaboração do discurso e, ao término, solicitado ao sujeito que o iniciasse, com a saída da assistente de pesquisa da sala. Transcorrido os quatro minutos, solicitou-se que o sujeito permanecesse no ambiente deitado, procurando relaxar, encerrando-se a sessão experimental. As sessões foram filmadas para posterior análise do desempenho dos sujeitos. Enquanto o sujeito proferia seu discurso frente à câmara, seu desempenho era simultaneamente transmitido na tela da televisão, posicionada também a sua frente, para que pudesse acompanhar o seu desempenho e, assim, aumentar a veracidade da situação.

\subsubsection{Codificação e Tratamento dos Dados}

\subsubsection{Proposição do Protocolo de Registro para a Análise das Filmagens Realizadas.}

Em um primeiro momento, procedeu-se ao levantamento de estudos empíricos relativos à temática "avaliação dos marcadores comportamentais de ansiedade" com base em uma busca sistemática na literatura recente, usando os indexadores Lilacs, Scielo, Medline e PsyclNFO, por meio do cruzamento das combinações possíveis entre as seguintes palavras-chave: behavioral assessment, social performance, performance, social skills, public-speaking, speech, 
speech disturbances, public speaking anxiety, simulated speech, anxiety, social phobia, social anxiety, e social anxiety disorder.

As categorias propostas inicialmente foram geradas a partir do exame de três fontes de informações, a saber: (a) artigos listados no procedimento de busca que se enquadravam no enfoque da temática mencionada anteriormente (HARB et al., 2003; LESNIAK-KARPIAK; MAZZOCCO; ROSS, 2003; SAWYER; BEHNKE, 2002; ZOHAR; LIVNE; FINE, 2003); (b) material já conhecido pelo pesquisador (AGUIAR, 2006; BAKER; EDELMANN, 2002; FYDRICH et al., 1998; HOFMANN et al., 1997; KNAPP, 1982; NORTON; HOPE, 2001; PEASE; PEASE, 2005; WENZEL et al., 2005); e (c) criação espontânea pelo pesquisador. Na leitura dos artigos arrolados, pretendeu-se identificar categorias em geral empregadas na codificação da ansiedade associada ao falar em público ou em situações típicas de interação social.

Em seguida, as categorias criadas e suas respectivas definições foram analisadas com outro pesquisador para a tomada de decisão acerca de sua pertinência e relevância para comporem o protocolo, considerando a natureza da tarefa desempenhada pelos participantes no estudo em questão. Quando necessário, as definições propostas foram reformuladas de modo a permitir maior precisão e clareza, eliminado possíveis sobreposições de categorias e padronizando as definições.

Com base nestes passos, elaborou-se uma pró-forma do protocolo de registro e procedeu-se a uma testagem piloto em dois casos, visando à verificação de sua adequação para a categorização dos desempenhos observados e da necessidade de ajuste das categorias propostas. Desse processo, resultou o sistema de classificação de categorias utilizado nesse estudo para a análise dos dados coletados. As categorias criadas para o protocolo de registro foram definidas e agrupadas em três classes de marcadores comportamentais, a saber: (a) marcadores verbais; (b) marcadores paralingüísticos; e (c) marcadores não-verbais que compreenderam as posturas corporais, gestos e expressões faciais apresentadas pelos sujeitos.

Cada definição proposta descreve um desempenho, verbal e/ou não verbal, específico exibido pelos sujeitos. Como partes integrantes do protocolo, ainda constavam: (a) uma avaliação geral do desempenho de cada sujeito, ou seja, de sua habilidade de falar em público; e (b) o tempo de duração do discurso e de latência para responder (iniciar o discurso) apresentada por cada sujeito. As definições das categorias de análise propostas encontram-se a seguir.

\subsubsection{Definição das categorias comportamentais do TSFP}

\section{A. Marcadores verbais:}


A.1. Conteúdo negativo: ação verbal do sujeito de fazer declarações, relatos ou comentários negativos relacionados ao tema do discurso;

A.2. Repetição de conteúdo: ação verbal do sujeito de repetir alguma sentença ou assunto proferido anteriormente;

A.3. Descontinuidade da fala: ação verbal do sujeito de fazer declarações sem conexão alguma entre elas (entre uma fala e outra) ou de interromper sentenças (frases ou orações) no contínuo da tarefa, tornando-as sem sentido ou significado, refletindo assim um discurso menos contínuo e mais truncado;

A.4. Uso de jargões: ação verbal do sujeito em pronunciar expressões de uso informal e popular (exemplos: "né", "tipo assim", "sabe", "é tudo de bom", e outras expressões do gênero).

\section{B. Marcadores paralingüísticos:}

B.1. Pausas: ação não-verbal do sujeito de interromper o fluxo do discurso (episódio de silêncio) durante um intervalo igual ou superior a 3 segundos (pausas silenciosas), ou emitir sons de hesitação no transcorrer do discurso, tais como: "ãnnn...", "é-é-é...", "hummm", entre outros (pausas preenchidas);

B.2. Risos inapropriados: ação não-verbal do sujeito de abrir a boca em sentido horizontal, tornando os dentes visíveis e os lados (cantos) de sua boca dirigidos para cima sem coerência com o conteúdo verbal;

B.3. Velocidade da fala inapropriada: ação não-verbal do sujeito de falar excessivamente rápido ou devagar ou com uma velocidade variante (alterna velocidade de fala apropriada e inapropriada);

B.4. Intensidade da voz inapropriada: ação não-verbal do sujeito de falar com um volume de voz excessivamente baixo (é ligeiramente ouvido) ou alto (pode causar algum desconforto auditivo para o ouvinte) ou volume variante (alterna intensidade de voz apropriada e inapropriada).

\section{Marcadores não-verbais (posturas corporais, gestos e expressões faciais):}

C.1. Evitação da tarefa: ação não-verbal do sujeito de permanecer em silêncio (sem verbalizar qualquer palavra ou frase) durante o tempo todo, não se engajando na execução da tarefa para a qual foi 
instruído, ou durante um intervalo de tempo igual ou superior a 20 segundos, contando após sinal dado pela auxiliar de pesquisar para dar início ao discurso;

C.2. Rigidez corporal: ação não-verbal do sujeito de sentar-se tenso ou rígido, com mudanças mínimas nas posições corporais, com a seguinte variação: agarrar firmemente os braços da cadeira com as mãos;

C.3. Inquietação: ação não-verbal do sujeito de mudar freqüente ou abruptamente as posições corporais, tais como balançar-se ou inclinar-se (curvar-se) em direções diferentes; ou emitir movimentos pequenos e irrequietos (movimentos repetidos das extremidades superiores de maneira rápida e involuntária), incluindo: contorcer as mãos, roçar um dedo sobre o outro ou sobre o braço da poltrona, enrolar os cabelos, brincar com as roupas ou quaisquer outros objetos de uso pessoal (por exemplo, presilha de cabelo), balançar o joelho ou uma das pernas e/ou bater levemente o pé (no mínimo três movimentos de vai-e-vem);

C.4. Atitude de intenção: ação não-verbal do sujeito de sentar-se na metade anterior da cadeira ou inclinar o tronco para frente com as mãos no joelho ou agarrando a cadeira com as duas mãos;

C.5. Desvio do olhar: ação não-verbal do sujeito de interromper o comportamento de olhar em direção à câmara ou evitar completamente esta resposta durante um intervalo igual ou superior a 10 segundos;

C.6. Movimentos faciais de desconforto: ação não-verbal do sujeito de morder os lábios ou franzir a testa;

C.7. Barreiras: ação não-verbal do sujeito de apresentar o comportamento de cruzar os membros superiores ou inferiores (braços ou pernas), mantendo-os um sobre o outro; ajustar a pulseira do relógio; bater ou esfregar as mãos, ou mesmo juntá-las ficando com os dedos entrelaçados; ou fazer quaisquer outros movimentos que deixem o braço atravessado na frente do corpo.

\section{Avaliação geral do desempenho}

Trata-se de uma avaliação (impressão geral) subjetiva do observador em relação à habilidade de falar em público exibida pelo sujeito em uma escala de 0 (extremamente inadequada) a 10 (muito adequada).

\section{E. Duração total do discurso e latência para responder:}


E.1. Latência da resposta: tempo despendido pelo sujeito em silêncio (sem verbalizar qualquer palavra ou frase) para dar início à tarefa após sinal dado pela auxiliar de pesquisa, ou seja, o tempo médio (em segundos) entre o término da resposta da auxiliar de pesquisa e o início da resposta do participante;

E.2. Tempo total de fala: duração total de tempo despendido pelo sujeito falando, permanecendo engajado na tarefa para a qual foi instruído;

\subsubsection{Procedimento de categorização e análise das sessões experimentais do TSFP}

O procedimento de categorização e análise das sessões experimentais seguiu os passos relatados a seguir.

Primeiramente, o conteúdo das filmagens foi observado e analisado pelo primeiro autor que desconhecia o grupo de origem dos sujeitos. A partir das filmagens de cada sujeito, uma série de ações foi conduzida, a saber: (a) verificaram-se as ocorrências dos marcadores comportamentais, denominados de categorias, registrando-se as suas frequências; (b) realizou-se uma avaliação geral da sua habilidade de falar em público; e (c) computaram-se a duração total do seu discurso e de sua latência para responder. Todas estas ações foram realizadas utilizando-se o protocolo de registro.

A avaliação geral da habilidade de falar em público, exibida por cada sujeito, foi feita em uma escala variando de 0 (extremamente inadequada) a 10 (muito adequada). Para efeitos de classificação do desempenho, a escala proposta para a avaliação da habilidade de falar em público apresentada pelo sujeito em questão seguiu a seguinte gradação:

- $0-2,5=$ carência de habilidades sociais apropriadas;

- 2,6 - 5 = nível mínimo de habilidades sociais apropriadas;

- 5,1 - 7,5 = nível moderado de habilidades sociais apropriadas;

- 7,6 - 10 = nível alto de habilidades sociais apropriadas.

O conteúdo do discurso dos sujeitos foi transcrito manualmente e depois digitado para possibilitar a análise e categorização dos marcadores verbais, principalmente da categoria descontinuidade da fala, devido à influência de vários fatores que atuaram no ambiente durante a coleta de dados e que, posteriormente, impossibilitariam o acompanhamento do fluxo contínuo da fala dos sujeitos. Estes fatores constituíram-se de: (a) ruído produzido pelo próprio equipamento de filmagem; (b) ruídos externos ao laboratório, onde o trabalho de coleta foi realizado; e (c) características vocais idiossincráticas de alguns sujeitos, como por exemplo, velocidade da voz excessivamente rápida ou intensidade de voz excessivamente baixa, 
que demandavam retroceder o vídeo várias vezes para a compreensão do que estava sendo dito.

Com relação à avaliação geral do desempenho dos sujeitos, procurouse levar em consideração todos os elementos analisados até o momento antes de realizá-la, ou seja, a presença e freqüência de todos os marcadores comportamentais de ansiedade levantados, assim como a duração total do discurso e a latência para iniciá-lo, além de uma comparação de desempenho entre os sujeitos.

Quanto à análise independente das 10 sessões de observação (38,5\% do total) pelos dois avaliadores externos, os índices gerais de concordância obtidos foram de $82,4 \%$ com o primeiro avaliador, e $83 \%$ com o segundo avaliador.

\subsubsection{Análise estatística dos dados coletados}

Para a comparação entre os grupos, consideraram-se a freqüência de ocorrência dos marcadores comportamentais e suas respectivas classes, a avaliação geral do desempenho, a duração do discurso e a latência para responder apresentada pelos sujeitos. Procedeu-se a comparação por meio do Teste de Mann-Whitney, adotando-se o nível de significância de $p \leq 0,05$.

Quanto à avaliação geral do desempenho exibido pelos sujeitos no TSFP, computou-se o percentual de sujeitos, agrupados como caso e não-caso, em cada nível de classificação do repertório de habilidades sociais de falar em público.

Em concordância com outro pesquisador, foram selecionados os itens do IHS-Del-Prette que melhor se ajustassem à habilidade de falar em público, a saber: itens 9 ("Evito fazer exposições ou palestras a pessoas desconhecidas"), 11 ("Em uma sala de aula ou reunião, se o professor ou dirigente faz uma afirmação incorreta, eu expresso meu ponto de vista"), 14 ("Faço exposição (por exemplo, palestras) em sala de aula ou no trabalho, quando sou indicado(a)"), 16 ("Em um grupo de pessoas conhecidas, se não concordo com a maioria, expresso verbalmente minha discordância") e 29 ("Na escola ou no trabalho, quando não compreendo uma explicação sobre algo que estou interessado(a), faço as perguntas que julgo necessárias ao meu esclarecimento"). Estes itens tiveram em comum o componente de falar e/ou se expor em público, alguns deles com possibilidade de rejeição, réplica, ou de oposição por parte do(s) interlocutor(es). Nas análises conduzidas, estes itens foram também somados para se obter um escore geral referente à habilidade de falar em público.

O Teste de Mann-Whitney foi empregado para a comparação dos grupos em relação a esses itens, ao escore geral da habilidade de falar em público, e ao escore total do IHS-Del-Prette, adotando-se o nível de significância de $p \leq 0,05$. 
Posteriormente, executaram-se correlações bivariadas de Spearman entre todas as categorias de análise (marcadores comportamentais e suas respectivas classes; avaliação geral do desempenho, incluindo a sua classificação; latência da resposta; e tempo total de fala) e os itens específicos do falar em público, assim como entre as categorias de análise e o escore geral da habilidade de falar em público. Devido ao caráter exploratório dessas análises e tamanho pequeno da amostra, apenas foram consideradas as correlações que demonstraram significância estatística $(p \leq 0,05)$ e sentido de conteúdo.

\section{Resultados}

\subsection{Caracterização da amostra}

Observou-se um predomínio do gênero feminino tanto na amostra total $(\mathrm{N}=16)$ como dentro de cada grupo $(\mathrm{N}=8)$ e, portanto, um percentual igual de sujeitos do mesmo gênero na composição dos dois grupos, não havendo diferença significativa entre eles em relação a esta variável $\left(\chi_{(1)}^{2}=0,00, p=1,00\right)$. A idade média dos sujeitos foi de 21,2 anos tanto para a amostra total como para os grupos caso e não-caso ( $D P=1,7 ; 1,3$; e 2,1, respectivamente).

\subsection{Comparação dos grupos caso e não-caso de TAS em relação aos marcadores comportamentais de ansiedade}

Para a comparação dos grupos quanto à frequência total de cada um dos marcadores comportamentais e suas respectivas classes, avaliação geral do desempenho, tempo total de fala e latência da resposta, procedeu-se o Teste Não-Paramétrico de Mann-Whitney, cujos resultados são apresentados na Tabela 1. 
Tabela 1 - Comparação dos grupos caso $(N=13)$ e não-caso de TAS ( $N=13$ ) em relação aos marcadores comportamentais de ansiedade e suas respectivas classes, avaliação geral do desempenho, duração total do discurso e latência da resposta.

\begin{tabular}{|c|c|c|}
\hline Categorias e Classes Avaliadas & $\mathrm{U}$ de Mann-Whitney & Probabilidade \\
\hline Classe de marcadores verbais & 56,50 & $p=0,151^{(\mathrm{NS})}$ \\
\hline Classe de marcadores paralingüísticos & 63,50 & $p=0,281^{(\text {NS })}$ \\
\hline Classe de marcadores não-verbais & 39,50 & $p=0,021^{*}$ \\
\hline Conteúdo negativo & 76,50 & $p=0,680^{(\text {NS })}$ \\
\hline Repetição de conteúdo & 50,50 & $p=0,073^{(\text {NS) }}$ \\
\hline Descontinuidade da fala & 71,50 & $p=0,503^{(\mathrm{NS})}$ \\
\hline Uso de jargões & 54,00 & $p=0,116^{(\mathrm{NS})}$ \\
\hline Pausas & 51,50 & $p=0,089^{(\mathrm{NS})}$ \\
\hline Risos inapropriados & 82,50 & $p=0,911^{(\mathrm{NS})}$ \\
\hline Velocidade de fala inapropriada & 48,00 & $p=0,057^{(\text {NS })}$ \\
\hline Intensidade de voz inapropriada & 60,50 & $p=0,199^{(\text {NS })}$ \\
\hline Evitação da tarefa & 60,00 & $p=0,088^{(\mathrm{NS})}$ \\
\hline Rigidez corporal & 72,00 & $p=0,385^{(\mathrm{NS})}$ \\
\hline Inquietação & 83,50 & $p=0,959^{(\text {NS })}$ \\
\hline Atitude de intenção & 80,50 & $p=0,816^{(\mathrm{NS})}$ \\
\hline Desvio do olhar & 59,00 & $p=0,110^{(\text {NS) }}$ \\
\hline Movimentos faciais de desconforto & 29,50 & $p=0,005^{*}$ \\
\hline Barreiras & 76,00 & $p=0,662^{(\text {NS) }}$ \\
\hline Avaliação geral do desempenho & 61,00 & $p=0,226^{(\text {NS })}$ \\
\hline Latência da resposta & 79,00 & $p=0,775^{(\mathrm{NS})}$ \\
\hline Tempo total de fala & 65,50 & $p=0,328^{\text {(NS) }}$ \\
\hline
\end{tabular}

(NS) $=$ diferença não significativa; ${ }^{*}=$ diferença significativa

Observou-se que os grupos caso e não-caso demonstraram diferenças significativas apenas em relação a movimentos faciais de desconforto e à classe de marcadores não-verbais, a qual essa mesma categoria pertence. Para essa categoria e sua classe, os postos médios do grupo não-caso $(17,73$ e 16,96$)$ foram maiores que os postos médios do grupo caso $(9,27$ e 10,04$)$. O grupo caso também não diferiu significativamente do grupo não-caso quanto aos tipos de pausa apresentados. Para as pausas preenchidas, o $U$ de Mann-Whitney foi 50,50 com um valor de probabilidade associada de 0,080 , e para as pausas silenciosas 56,00, com um valor de probabilidade associada de 0,120 .

\subsection{Avaliação do repertório de habilidades sociais de falar em público dos grupos}

Para efeitos de avaliação do desempenho exibido pelos sujeitos no TSFP, a Figura 1 apresenta o percentual de sujeitos, agrupados como 
caso e não-caso de TAS, em cada nível de classificação do repertório de habilidades sociais de falar em público.

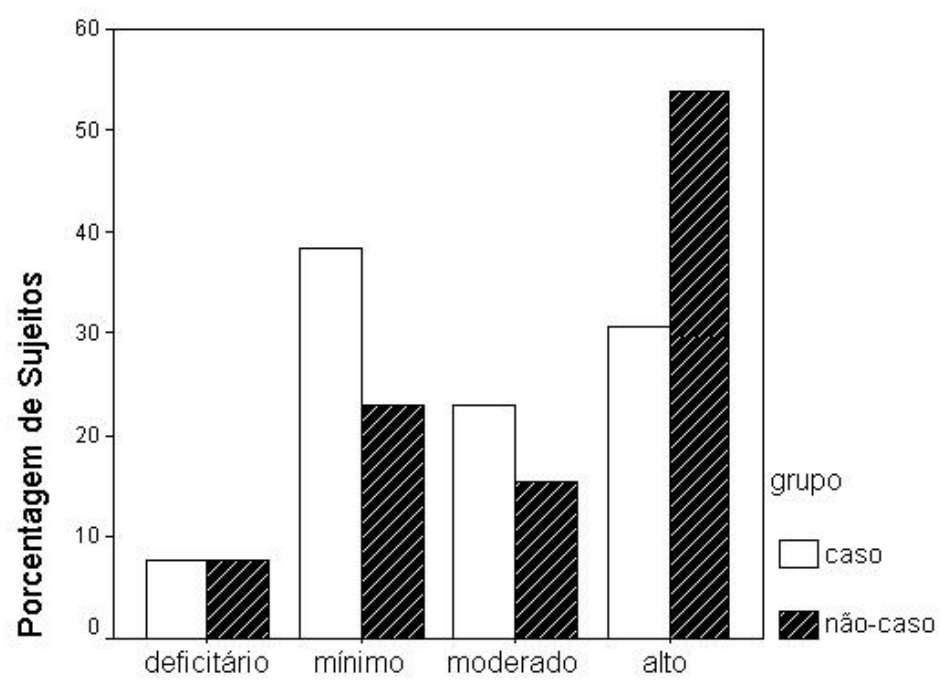

classificação do repertório de habilidades sociais

Figura 1 - Classificação do repertório de habilidades sociais de falar em público

demonstrado pelos sujeitos dos grupos caso $(N=13)$ e não-caso de TAS $(N=13)$.

Os dois grupos apresentaram o mesmo percentual de sujeitos $(7,7 \%)$ com carência de habilidades sociais apropriadas para falar em público ( $\mathrm{N}=1$ para os dois grupos), ou seja, com um repertório deficitário. $38,4 \%$ do grupo caso $(\mathrm{N}=5)$ e $23,1 \%$ do grupo não-caso $(\mathrm{N}=3$ ) exibiram um nível mínimo de habilidades sociais apropriadas. No nível moderado, observaram-se $23,1 \%$ do grupo caso $(\mathrm{N}=3)$ e $15,4 \%$ do grupo não-caso $(\mathrm{N}=2$ ). Em contrapartida, $30,8 \%$ do grupo caso ( $\mathrm{N}$ $=4)$ e $53,8 \%$ do grupo não-caso $(N=7)$ demonstraram um nível alto de habilidades sociais apropriadas. No geral, $69,2 \%$ do grupo não-caso foi avaliado como apresentando um nível de habilidades sociais variando de moderado a alto em comparação a $53,9 \%$ do grupo caso.

Os grupos caso e não-caso de TAS foram ainda comparados em relação ao escore total do IHS-Del-Prette, aos itens específicos deste instrumento referentes à habilidade de falar em público, e ao escore geral obtido para esta habilidade, cujos dados são apresentados na Tabela 2. 
Tabela 2 - Comparação dos grupos caso $(N=13)$ e não-caso de TAS $(N=12)$ em relação aos itens do IHS-Del-Prette indicativos da habilidade de falar em público e ao escore geral desta habilidade.

\begin{tabular}{lcl}
\hline \multicolumn{1}{c}{ Habilidades de falar em público } & U & Probabilidade \\
\hline Item 9 (falar a público desconhecido) & 13,00 & $p<0,001^{*}$ \\
Item 11 (discordar de autoridade) & 29,00 & $p=0,005^{*}$ \\
Item 14 (falar a público conhecido) & 39,50 & $p=0,031^{*}$ \\
Item 16 (discordar do grupo) & 31,50 & $p=0,008^{*}$ \\
Item 29 (fazer perguntas a conhecidos) & 56,00 & $p=0,216^{\text {(NS) }}$ \\
Escore geral da habilidade de falar em público & 18,00 & $p=0,001^{*}$ \\
\hline
\end{tabular}

$\mathrm{U}=\mathrm{U}$ de $M a n n-W h i t n e y ;{ }^{*}=$ diferença significativa; (NS) = diferença não significativa

Os grupos diferiram significativamente em quatro dos cinco itens indicativos da habilidade de falar em público (item 9, 11, 14, e 16), inclusive em relação ao escore geral da habilidade de falar em público. Para esses itens e escore geral, os postos médios do grupo caso $(8,00,9,23,10,04,9,42$ e 8,38 , respectivamente) foram menores que os postos médios do grupo não-caso $(18,42,17,08$, $16,21,16,88$ e 18,00, respectivamente).

Quanto ao escore total do IHS-Del-Prette, foi encontrada uma diferença significativa entre os grupos $(U=2,50, p<0,001)$, com 0 posto médio do grupo caso $(7,19)$ também sendo menor do que aquele evidenciado pelo grupo não-caso $(19,28)$.

\subsection{Habilidades sociais de falar em público e marcadores comportamentais de ansiedade}

Todas as categorias de análise (marcadores comportamentais e suas respectivas classes; avaliação geral do desempenho, incluindo a sua classificação; latência da resposta; e tempo total de fala) foram correlacionadas com os itens específicos do falar em público e também com o escore geral da habilidade de falar em público, extraídos do IHS-Del-Prette. Como dito anteriormente, devido ao caráter exploratório dessas análises e tamanho pequeno da amostra, foram consideradas apenas as correlações que demonstraram significância estatística $(p \leq 0,05)$ e sentido de conteúdo. Esses dados são apresentados na Tabela 3. 
Tabela 3 - Correlações significativas encontradas entre as categorias de análise e as habilidades de falar em público na amostra do estudo $(\mathrm{N}=25)$.

\begin{tabular}{lrc}
\hline \multicolumn{1}{c}{ Correlações significativas } & $\rho$ & $\boldsymbol{p}$ \\
\hline - Evitação da tarefa x item 9 (falar a público desconhecido) & $-0,43$ & 0,031 \\
- Evitação da tarefa x item 14 (falar a público conhecido) & $-0,63$ & 0,001 \\
- Evitação da tarefa x escore geral da HSFP & $-0,45$ & 0,023 \\
- Desvio do olhar x item 14 (falar a público conhecido) & $-0,42$ & 0,039 \\
- Desvio do olhar x escore geral da HSFP & $-0,49$ & 0,014 \\
- Tempo total de fala x item 14 (falar a público conhecido) & 0,64 & 0,001 \\
- Tempo total de fala x escore geral da HSFP & 0,45 & 0,025 \\
- Avaliação geral do desempenho x item 14 (falar a público conhecido) & 0,51 & 0,009 \\
- Avaliação geral do desempenho x escore geral da HSFP & 0,49 & 0,013 \\
- Classificação do desempenho x item 11 (discordar de autoridade) & 0,46 & 0,020 \\
- Classificação do desempenho x item 14 (falar a público conhecido) & 0,52 & 0,007 \\
- Classificação do desempenho x escore geral da HSFP & 0,54 & 0,006 \\
\hline
\end{tabular}

$\rho$ de Spearman; $p=$ probabilidade associada; HSFP $=$ habilidade social de falar em público

Todas as correlações com significância estatística e de conteúdo encontradas variaram de 0,42 a 0,64, ou seja, de moderada a alta, independentemente do relacionamento entre as variáveis ser positivo ou negativo. O item 14 (falar a público conhecido) e o escore geral da habilidade de falar em público foram as variáveis que mais se correlacionaram com aquelas referentes às categorias de análise do TSFP. Estas correlações indicaram que quanto maior a habilidade de falar a um público conhecido ou a habilidade geral de falar em público de um indivíduo, ele demonstrará menos evitação de tarefa, menor frequência de desvio de olhar, um melhor aproveitamento do tempo disponível e, consequentemente, será avaliado mais positivamente pela sua audiência. Menos evitação de tarefa esteve também associado a uma maior habilidade de falar a público desconhecido (item 9); e maior frequência da habilidade de discordar de autoridade em situações sociais (item 11) associada a uma melhor avaliação do desempenho. Por exemplo, como o próprio item 11 sugere, estas situações sociais seriam ou uma sala de aula ou uma reunião.

\section{Discussão}

As poucas diferenças significativas entre os grupos caso e não-caso de TAS em relação à maioria das categorias e suas respectivas classes podem ser atribuídas a dois fatores. O primeiro deles, de caráter metodológico, refere-se ao fato dos sujeitos diagnosticados com TAS para compor o grupo caso não terem sido avaliados quanto 
aos subtipos de manifestação do transtorno, ou seja, se generalizado ou circunscrito. Desse modo, os sujeitos com TAS que participaram deste estudo poderiam se enquadrar no subtipo circunscrito ou específico, temendo apenas uma ou poucas situações públicas de desempenho, com exceção de falar em público, tais como comer, beber, tocar um instrumento na frente dos outros ou usar banheiro público.

No entanto, mesmo que esses indivíduos apresentassem o medo de falar em público pregressamente, pode-se pensar que as exigências acadêmicas de apresentação de trabalhos e seminários em sala de aula ou mesmo exposição de opiniões em grupo, tão frequentes na vida universitária, podem ter gradualmente reduzido as reações mais severas e debilitantes de ansiedade frente a esse tipo de tarefa. A exposição dos sujeitos aos estímulos ansiogênicos dessas situações pode ter ocorrido a custos bastante altos para eles, mas ao mesmo tempo é possível que tenha contribuído para que enfrentassem de forma relativamente mais adaptativa os seus medos.

Em um estudo que mediu a reatividade fisiológica para situações temidas (BOONE et al., 1999), os indivíduos com TAS específico apresentaram freqüência cardíaca mais alta em uma tarefa de falar em público, do que o subtipo generalizado. Este estudo também examinou as respostas do TAS generalizado e específico em um roleplay de interação social (conversar com um desconhecido do gênero oposto). Este tipo de situação não produziu respostas fisiológicas diferenciais, ao contrário da diferença encontrada para o role-play de falar em público. Em seu estudo, Tran e Chambless (1995) observaram que o grupo com TAS específico relatou maior ansiedade comparado ao grupo com TAS generalizado sem transtorno de personalidade evitativa somente durante o discurso. Como defendem Hook e Valentiner (2002), estes resultados são consistentes com a concepção de que o TAS específico implica em ansiedade de desempenho e sugere que reações de ansiedade aguda associadas ao TAS específico não ocorrem durante desafios de interação social.

Um outro fator diz respeito à possibilidade dos sujeitos com TAS apresentarem um bom processamento cognitivo para alguns estímulos externos, sendo assim capazes de discriminar que estavam se comportando frente a uma câmara e não frente a uma audiência de pessoas, mesmo sendo a situação em si ansiogênica. Desta forma, essa capacidade discriminativa pode ter feito com que a situação tivesse um menor impacto sobre os seus desempenhos. Conseqüentemente, surge uma questão básica a esse respeito que não pode ser respondida por este estudo: 0 desempenho exibido durante a situação experimental do TSFP corresponderia ao desempenho observado em uma situação natural de falar em público? Dado que o uso de métodos observacionais análogos é universal em avaliação comportamental, torna-se imperativo que novas pesquisas 
sejam conduzidas para abordar essa questão quanto à validade ecológica, ou seja, o grau em que as condições de um estudo são verdadeiras ou aplicáveis para a vida real, dimensionando assim a possibilidade de generalização dos resultados.

Este estudo empregou níveis de medida comportamental tanto molecular quanto molar do desempenho dos sujeitos. O nível molecular consistiu em avaliações altamente específicas usando a frequência e/ou duração de comportamentos específicos, enquanto o nível molar compreendeu avaliações mais gerais, uma usando a frequência das classes de comportamentos e outra realizada sobre uma escala. A avaliação no nível molecular dos marcadores comportamentais de ansiedade forneceu informações importantes acerca de pontos fracos específicos dos indivíduos avaliados que podem ser usadas tanto no planejamento de tratamentos quanto durante o curso de tratamentos voltados para reduzir déficits e promover desempenhos mais habilidosos.

Dentro desta perspectiva, o conteúdo das filmagens do TSFP poderia ser empregado como técnica de vídeofeedback acerca do desempenho dos sujeitos em programas de Treinamento em Habilidades Sociais voltado para o contexto acadêmico, visando a modelagem de desempenhos mais adequados. Além disso, esse conteúdo pode fornecer feedback corretivo importante aos sujeitos com TAS, uma vez que eles podem crer que se comportam mais pobremente do que de fato se comportam. Nesta direção, os resultados de Rapee e Hayman (1996) indicam que quando indivíduos socialmente ansiosos avaliam o próprio desempenho em um discurso por meio do registro em vídeo, eles são capazes de avaliá-lo de maneira mais consistente com a avaliação de observadores independentes. Os sujeitos que não foram expostos a esse registro, tenderam a subestimar o próprio desempenho em comparação com avaliações fornecidas por observadores.

Considerou-se a categoria movimentos faciais de desconforto como uma medida pouco válida do desempenho dos sujeitos à medida que a sua definição contemplou toda e qualquer forma de franzir a testa e morder os lábios, tornando-a imprecisa. Por exemplo, franzir a testa, erguendo as sobrancelhas, acompanha muito do que é expresso verbalmente. Além disso, nem sempre foi possível identificar claramente, pela morfologia do gesto, se morder os lábios tinha a função de mordida mesmo ou de umedecer os lábios. Portanto, ou essa categoria precisaria ser revista para garantir uma melhor definição ou a análise do conteúdo das filmagens deveria contar com equipamentos que possibilitassem uma visualização mais clara e precisa daquilo que estava sendo observado.

Com relação aos itens indicativos da habilidade social de falar em público, selecionados a partir do IHS-Del-Prette, a maioria (item 9, 11,14 e 16) evidenciou significativamente distinguir os sujeitos do 
grupo caso daqueles do grupo não-caso de TAS, com exceção apenas do item 29. Além disso, o escore geral da habilidade de falar em público, obtido pela somatória dos itens, mostrou coerentemente discriminar ambos os grupos.

Possivelmente, o contraste encontrado entre a capacidade discriminativa desses itens e os marcadores comportamentais de ansiedade seja atribuído ou explicado pelos diferentes tipos de déficits avaliados em cada situação específica. No primeiro caso, os itens do IHS-Del-Prette avaliam, assumidamente, os déficits de desempenho existentes no repertório de um indivíduo e, no segundo, pode-se dizer que os marcadores comportamentais de ansiedade avaliaram os déficits de fluência dos sujeitos. Sendo assim, ambos os instrumentos, o IHS e o TSFP, avaliam dimensões distintas do repertório comportamental de um indivíduo.

\section{Conclusões}

Apesar das limitações deste estudo, o TSFP mostrou-se um procedimento bastante útil para avaliar os sinais ou marcadores comportamentais de ansiedade à medida que simula uma das situações sociais temidas mais prevalente nos diferentes subtipos do TAS, como também na população geral. Desse modo, a situação experimental do TSFP demonstrou que a ansiedade experimentada pelos indivíduos em situações de falar em público é manifestada comportalmente e pode ser confiavelmente avaliada. Além disso, o TSFP, por ser uma tarefa de avaliação padronizada, facilita comparações acerca do desempenho de indivíduos ou grupos definidos.

Os resultados deste estudo apontam para a possibilidade do TSFP ser usado em programas de Treinamento em Habilidades Sociais no contexto educacional para a avaliação tanto dos recursos quanto dos déficits comportamentais de habilidades sociais de falar em público, visando promover um melhor desempenho de estudantes universitários nas atividades acadêmicas de apresentação de trabalhos e seminários. Esse refinamento no repertório comportamental dos alunos refletiria, futuramente, em uma melhor qualificação profissional dos mesmos para enfrentar o mercado de trabalho.

Um limite deste estudo foi não ter sido tomada uma medida dos sintomas de ansiedade ao longo do procedimento do TSFP e associála tanto aos marcadores comportamentais de ansiedade propostos quanto às habilidades sociais de falar em público extraídas do IHSDel-Prette, visando explorar as possíveis relações entre os relatos subjetivos e as manifestações comportamentais de ansiedade e ainda entre estes relatos e a habilidade de falar em público dos sujeitos. 
A análise dos resultados deste estudo aponta para a necessidade de novos estudos com amostras não-clínica e clínicas mais amplas, de indivíduos com TAS dos subtipos generalizado e circunscrito, com avaliação prévia dos sujeitos quanto ao medo de falar em público, dando assim respaldo para comparações mais consistentes entre os grupos, e que contemplem a validade ecológica e possibilite uma generalização mais ampla dos resultados.

\section{Referências}

AGUIAR, A. A. R. Construção e avaliação de um programa multimodal de habilidades comunicativas junto a adultos com deficiência mental. 2006. 201f. Tese (Doutorado em Educação Especial) - Universidade Federal de São Carlos, São Paulo.

BAKER, S. R.; EDELMANN, R. J. Is social phobia related to lack of social skills? Duration of skill-related behaviours and ratings of behavioural adequacy. British Journal of Clinical Psychology, London, v. 41, n. 3, p. 243-257, Sept. 2002.

BOONE, M. L.; MCNEIL, D. W.; MASIA, C. L.; TURK, C. L.; CARTER, L. E.; RIES, B. J.; LEWIN, M. R. Multimodal comparisons of social phobia subtypes and avoidant personality disorder. Journal of Anxiety Disorders, Oxford, v. 13, n. 3, p. 271-292, May 1999.

CHAVES, D. I. Efeito da ocitocina sobre a ansiedade experimental em voluntários saudáveis. 2007. 84f. Dissertação (Mestrado em Toxicologia) - Faculdade de Ciências Farmacêuticas de Ribeirão Preto, Universidade de São Paulo, Ribeirão Preto, São Paulo. CONNOR, K. M.; KOBAK, K. A.; CHURCHILL, L. E.; KATZELNICK, D.; DAVIDSON, J. R. T. Mini-SPIN: a brief screening assessment for generalized social anxiety disorder. Depression and Anxiety, New York, v. 14, n. 2, p. 137-140. 2001.

CRIPPA J. A. S.; OSÓRIO, F. L.; DEL-BEN, C. M.; FILHO, A. S.; FREITAS, M. C. S.; LOUREIRO, S. R. Comparability between telephone and face-toface structured clinical interview for DSM-IV in assessing social anxiety disorder. Perspectives in Psychiatric Care, Hillsdale, v. 44, n. 4, p. 241-247, 2008.

DEL-BEN, C. M.; VILELA, J. A. A.; CRIPPA, J. A. S.; HALLAK, J. E. C.; LABATE, C. M.; ZUARDI, A. W. Confiabilidade da entrevista clínica estruturada para o DSM-IV - versão clínica traduzida para o português. Revista Brasileira de Psiquiatria, São Paulo, v. 23, n. 3, p. 156-159, Set. 2001a.

DEL-BEN, C. M.; VILELA, J. A. A.; HETEM, L. A. B.; GUIMARÃES, F. S.; GRAEFF, F. G.; ZUARDI, A.W. Do panic patients process unconditioned fear vs. Conditioned anxiety differently than normal subjects? Psychiatry Research, London, v. 104, n. 3, p. 227-237, 2001b. 
DEL PRetTe, A.; DEL PReTte, Z. A. P.; CASTElO BRANCO, U. V. Competência social na formação do psicólogo. Paidéia: Cadernos de Psicologia e Educação, Ribeirão Preto, v. 2, n. 1, p. 40-50, Fev. $1992 a$.

DEL PRETTE, Z. A. P.; DEL PRETTE, A.; BARReTO, M. C. M. Análise de um inventário de habilidades sociais (IHS) em uma amostra de universitários. Psicologia: teoria e pesquisa, Brasília, v. 14, n. 3, p. 219-228, 1998.

DEL PRETTE, Z. A. P.; DEL PRETTE, A.; CASTElO BRANCO, U. V. Competência social: um levantamento de situações críticas de interação para estudantes de Psicologia. In: CONGRESSO NACIONAL DE PSICOLOGIA ESCOLAR, 1., 1992, Valinhos. Anais do I Congresso Nacional de Psicologia Escolar. Campinas: Abrapee/Atomo, 1992b, p. 384-387.

D'EL REY, G. J. F.; PACINI, C. A. Medo de falar em público em uma amostra da população: Prevalência, impacto no funcionamento pessoal e tratamento. Psicologia: Teoria e Pesquisa, Brasília, v. 21, n. 2, p. 237-242, 2005.

FYDRICH, T.; CHAMBLESS, D. L.; PERRY, K. J.; BUERGENER, F.; BEAZLEY, M. B. Behavioral assessment of social performance: a rating system for social phobia. Behaviour Research and Therapy, Oxford, v. 36, n. 10, p. 995-1010, Oct. 1998.

FURMARK, T.; TILLFORS, M.; EVERZ, P. -O.; MARTEINSDOTTIR, I.; GEFVERT, O.; FREDRIKSON, M. Social phobia in the general population: prevalence and sociodemographic profile. Social Psychiatry and Psychiatric Epidemiology, Berlin, v. 34, n. 8, p. 416-424, Sept. 1999.

FURMARK, T.; TILLFORS, M.; STATTIN, H.; EKSELIUS, L.; FREDRIKSON, M. Social phobia subtypes in the general population revealed by cluster analysis. Psychological Medicine, London, v. 30, n. 6, p. 1335-1344, Nov. 2000.

GEER, J. H. The development of a scale to measure fear. Behaviour Research and Therapy, Oxford, v. 3, n. 1, p. 45-53, Aug. 1965.

GIRDER S. S.; TURNER, J. R.; SHERWOOD, A.; LIGHT, K. C. Gender differences in blood pressure control during a variety of behavioral stressores. Psychosomatic Medicine, Philadelphia, v. 52, n. 5, p.

571-591, 1990.

GONZALEZ-BONO, E.; MOYA-ALBIOL, L.; SALVADOR, A.; CARRILLO, E.; RICARTE, J.; GOMEZ-AMOR, J. Anticipatory autonomic response to a public speaking task in women: The role of trait anxiety. Biological Psychology, Amsterdam, v. 60, n. 1, p. 37-39, 2002.

GRAEFF, F. G. et al. Pharmacology of human experimental anxiety. Brazilian Journal of Medical and Biological Research, Ribeirão Preto, v. 36, n. 4, p. 421-432, Apr. 2003. 
GUIMARÃES, F. S.; MBAYA, O. S.; DEAKIN, J. F. W. Ritanserin facilitates anxiety in simulated public-speaking paradigm. J ournal of Psychopharmacology, Oxford, v.11, n. 3, p. 225-231, 1997.

GUIMARÃES, F. S.; ZUARDI, A. W.; GRAEFF, F. G. Effect of chlorimipramine and maprotiline on experimental anxiety in humans. J ournal of Psychopharmacology, Thousand Oaks, v. 1, n. 3, p. 184-192, 1988.

HARB, G. C.; ENG, W.; ZAIDER, T.; HEIMBERG, R. G. Behavioral assessment of public-speaking anxiety using a modified version of the Social Performance Rating Scale. Behaviour Research and Therapy, Oxford, v. 41, n. 11, p. 1373-1380, Nov. 2003.

HARRIS, S. P.; KEMMERLING, R. L.; NORTH, M. M. Brief virtual reality therapy for public speaking anxiety. Cyberpsychology \& Behavior, Larchmont, v. 5, n. 6, p. 543-550, Dec. 2002.

HETEM, L. A. B.; DE SOUZA, C. J.; GUIMARÃES, F. S.; ZUARDI, A. W.; GRAEFF, F. G. Effect of d-fenfluramine on human experimental anxiety. Psychopharmacology, Berlin, v. 127, n. 3, p. 276-282, Oct. 1996.

HOFMANN, S. G.; GERLACH, A. L.; WENDER, A.; ROTH, W. T. Speech disturbances and gaze behavior during public speaking in subtypes of social phobia. Journal of Anxiety Disorders, New York, v. 11, n. 6, p. 573-585, Nov./Dec. 1997.

HOOK, J. N.; VALENTINER, D. P. Are Specific and Generalized Social Phobias Qualitatively Distinct? Clinical Psychology: Science and Practice, Malden, v. 9, n. 4, Winter 2002.

KNAPP, M. La comunicación no verbal. Buenos Aires: Paidós, 1982.

LEE, J. M.; KIM, H.; OH, M. J.; KU, J. H.; JANG, D. P.; KIM, I. Y.; KIM, S. I. Development of a virtual speaking simulator using image based rendering. Studies in Health Technology and I nformatics, Amsterdam, v. 85, p. 258-260, 2002a.

LEE, J. M.; KU., J. H.; JANG, D. P.; KIM, D. H.; CHOI, Y. H.; KIM, I. Y.; KIM, S. I. Virtual reality system for treatment of the fear of public speaking using image based rendering and moving pictures. Cyberpsychology \& Behavior, Larchmont, v. 5, n. 3, p. 191-195, Jun. 2002b.

LESNI AK-KARPI AK, K.; MAZZOCCO, M. M. M.; ROSS, J. L. Behavioral assessment of social anxiety in females with Turner or Fragile $X$ Syndrome. Journal of Autism and Developmental Disorders, New York, v. 33, n. 1, p. 55-67, Feb. 2003.

MANNUZZA, S.; SCHNEIER, F. R.; CHAPMAN, T. F.; LIEBOWITZ, M. R.; KLEIN D. F.; FYER, A. J. Generalized social phobia: reliability and validity. Archives of General Psychiatry, Chicago, v. 52, n. 3, p. 230-237, Mar. 1995.

MCNAIR, D. M.; FRANKENTHALER, L. M.; CZERLINSKY, T.; WHITE, T. W.; SASSON, S.; FISHER, S. Simulated public speaking as a model of 
clinical anxiety. Psychopharmacology, Berlin, v. 77, n. 1, p. 7-10, May 1982.

NORTON, P. J.; HOPE, D. A. Analogue observational methods in the assessment of social functioning in adults. Psychological Assessment, Norwood, v. 13, n. 1, p. 59-72, Mar. 2001.

OSÓRIO, F. L.; CRIPPA, J. A.; LOUREIRO, S. R. Experimental models for the evaluation of speech and public speaking anxiety: a critical review of the designs adopted. Journal of Speech-Language Pathology and Applied Behavior Analysis, Philadelphia, n. 2.43.1, p. 97-121, Mar. 2008a. Special issue.

OSÓRIO, F. L.; CRIPPA, J. A.; LOUREIRO, S. R. Escala para autoavaliação ao falar em público (SSPS): Adaptação transcultural e consistência interna da versão brasileira. Revista de Psiquiatria Clínica, São Paulo, v. 35, n. 6, p. 207-211, 2008b.

PEASE, A.; PEASE, B. Desvendando os segredos da linguagem corporal. 3. ed. Tradução Pedro Jorgensen Junior. Rio de Janeiro: Sextante, 2005.

PERTAUB, D. P.; SLATER, M.; BARKER, C. An experiment on fear of public speaking in virtual reality. Studies in Health Technology and I nformatics, Amsterdam, v. 81, p. 372-378, 2001.

PHILLIPS, G. C.; J ONES, G. E.; RIEGER, E. R.; SNELL, J. B. Normative data for the personal report of confidence as a speaker. Journal of Anxiety Disorders, New York, v. 11, n. 2, p. 215-220, Mar./Apr. 1997.

RAPEE, R. M.; HAYMAN, K. The effects of video feedback on the selfevaluation of performance in socially anxious subjects. Behaviour Research and Therapy, Oxford, v. 34, n. 4, p. 315-322, Apr. 1996. RAPEE, R. M.; LIM, L. Discrepancy between self and observer ratings of performance in social phobics. Journal of Abnormal Psychology, Boston, v. 101, n. 4, p. 728-731, Nov. 1992.

SAAB, P. G.; MATTHEUS, K. A.; STONEY, C. M.; MCDONALD, R. H. Premenopausal and postmenopausal women differ in their cardiovascular and neuroendocrine responses to behavioral stressors. Psychophysiology, Baltimore, v. 26, n. 3, p. 270-280, May 1989.

SAWYER, C. R.; BEHNKE, R. R. Behavioral inhibition and the communication of public speaking state anxiety. Western J ournal of Communication, Salt Lake City, v. 66, n. 4, p. 412-422, Fall 2002. SPIELBERGER, C. D.; GORSUCH, R. L.; LUSHINE, R. E. Manual for the state-trait anxiety inventory. Palo Alto: Consulting Psychologists, 1970.

STEIN, M. B.; WALKER, J. R.; FORDE, D. R. Public-Speaking fears in a community sample: prevalence, impact on functioning and diagnostic classification. Archives of General Psychiatry, Chicago, v. 53, n. 2, p. 169-174, Feb. 1996.

TILLFORS, M.; FURMARK, T.; MARTEINSDOTTIR, I.; FREDRIKSON, M. Cerebral blood flow during anticipation of public speaking in social 
phobia: a PET study. Biological Psychiatry, Amsterdam, New York, v. 52, n. 11, p. 1113-1119, Dec. 2002.

TRAN, G. Q.; CHAMBLESS, D. L. Psychopathology of social phobia: effects of subtype and of avoidant personality disorder. Journal of Anxiety Disorders, Oxford, v. 9, n. 6, p. 489-501, Nov./Dec. 1995. WENZEL, A.; GRAFF-DOLEZAL, J.; MACHO, M.; BRENDLE, J. R. Communication and social skills in socially anxious and nonanxious individuals in the context of romantic relationships. Behaviour Research and Therapy, Oxford, v. 43, n. 4, p. 505-519, Apr. 2005. ZOHAR, D.; LIVNE, Y.; FINE, J. The effect of anxiety on linguistic parameters of public speech: a verbal impairment model. Anxiety, Stress and Coping, London, v. 16, n. 3, p. 293-306, Sept. 2003. ZUARDI, A. W.; KARNIOL, I. G. Estudo transcultural de uma escala de auto-avaliação para estados subjetivos. Jornal Brasileiro de Psiquiatria, Rio de Janeiro, v. 31, n. 5, p. 403-406, 1981.

ZUARDI, A. W.; COSME, R. A.; GRAEFF, F. G.; GUMARÃES, F. S. Effect of ipsapirone and cannabidiol on human experimental anxiety. J ournal of Psychopharmacology, Oxford, v. 7, n. 1, p. 82-88, Jan. 1993.

\section{Endereço para correspondência \\ Antonio Paulo Angélico}

Praça Dom Helvécio, 74, Dom Bosco, CEP: 36301-160, São João del-Rei - MG, Brasil

Endereço eletrônico: angelico@ufsj.edu.br

J osé Alexandre S. Crippa

Av. Bandeirantes, 3900, CEP 14049-900, Ribeirão Preto - SP, Brasil

Endereço eletrônico: jcrippa@fmrp.usp.br

Sonia Regina Loureiro

Rua Tenente Catão Roxo, 2650, CEP 14051-140, Ribeirão Preto-SP, Brasil

Endereço eletrônico: srlourei@fmrp.usp.br

Recebido em: $27 / 07 / 2010$

Reformulado em: 14/12/2010

Aceito para publicação em: 20/12/2010

Acompanhamento do processo editorial: Adriana Benevides Soares

\section{Notas}

* Professor Doutor do Departamento de Psicologia da Universidade Federal de São J oão del-Rei (UFSJ); Laboratório de Pesquisa em Saúde Mental (LAPSAM).

**Professor Doutor do Departamento de Neurociências e Ciências do Comportamento da Faculdade de Medicina de Ribeirão Preto da Universidade de São Paulo; Hospital das Clínicas.

***Professora Doutora do Departamento de Neurociências e Ciências do Comportamento da Faculdade de Medicina de Ribeirão Preto da Universidade de São Paulo; Programa de Pós-Graduação em Saúde Mental. 